\title{
Experimental and theoretical thermal analysis of CVD diamond window units for the ITER upper launcher
}

\author{
T. A. Scherera, R. Heidingera, A. Meier ${ }^{a}$, D. Strauss ${ }^{a}$, K. Takahashi ${ }^{b}$, K. Kajiwara ${ }^{\text {b }}$, K. Sakamoto ${ }^{b}$ \\ aInstitut für Materialforschung IMF-I, Association EURATOM-FZK, Forschungszentrum Karlsruhe, D-76021 Karlsruhe, Germany \\ b Plasma Heating Technology Group, Japan Atomic Energy Agency (JAEA), 801-1 Mukoyama, Naka, Ibaraki 311-0193 Japan
}

\begin{abstract}
An ITER torus window prototype with CVD diamond disks and corrugated waveguides are being investigated by using IR imaging and temperature measuring technique during high power RF microwave loading up to $1 \mathrm{MW}$ at a frequency of $170 \mathrm{GHz}$ at the JAEA gyrotron facility. To evaluate the cooling efficiency of the window design the temperature distribution over the diamond disk area is measured and compared with a theoretical thermal FEM analysis.
\end{abstract}
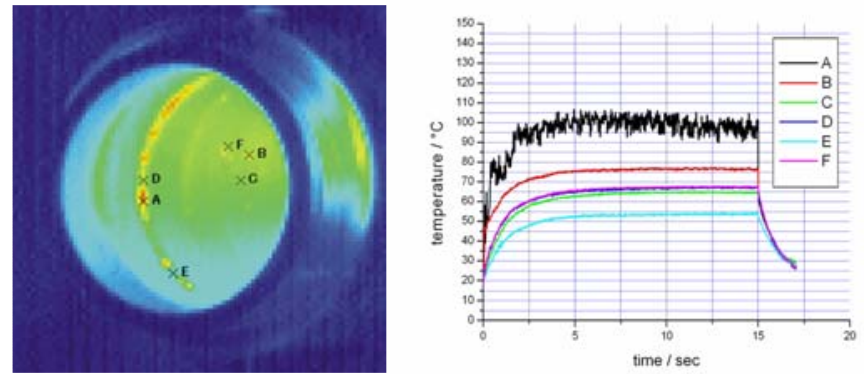

Fig. 1 IR picture (left) and temperature evolution (right) on a CVD diamond disk for a $320 \mathrm{~kW} / 15 \mathrm{sec}$ load (loss tangent: $3.4 \cdot 10^{-5}$ ).

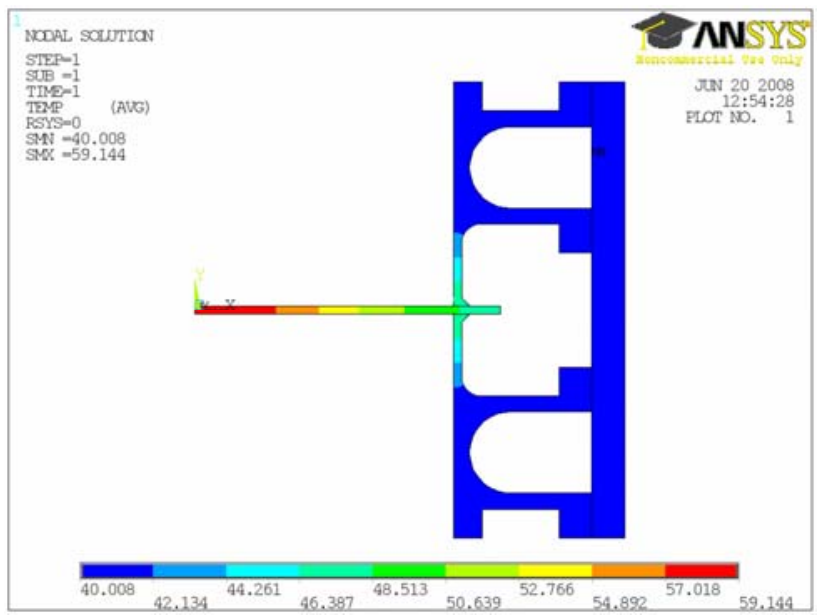

Fig. 2 Steady state ANSYS FEM analysis of the diamond window assembly for $320 \mathrm{~kW}$ microwave power.

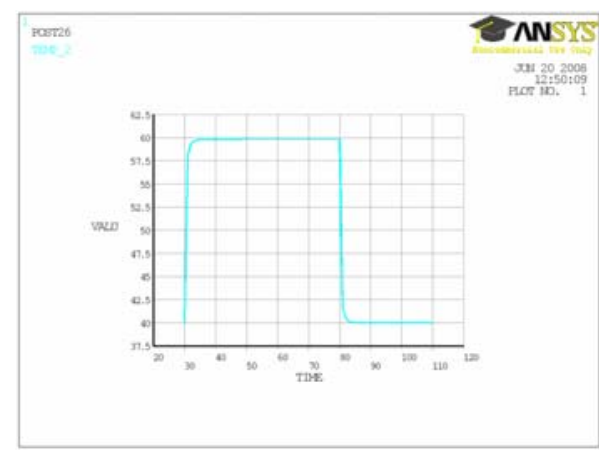

Fig. 3 Transient analysis of the diamond window assembly for $320 \mathrm{~kW} / 50 \mathrm{sec}$ microwave power.
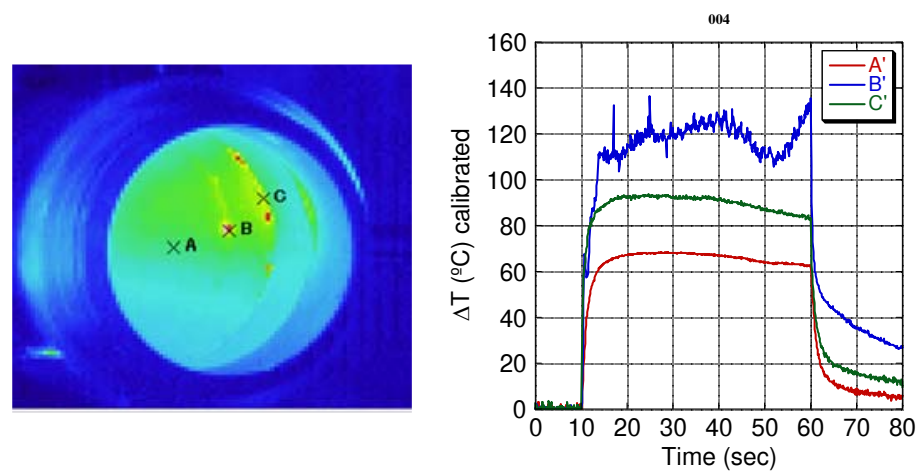

Fig. 4 Temperature-time dependence on a CVD diamond disk for a $320 \mathrm{~kW} / 50 \mathrm{sec}$ power and the corresponding IR picture. Additional heating by parasitic cavities (mode mixing) can be observed.

\section{Conclusion and outlook}

High power RF measurements at $170 \mathrm{GHz}$ have been performed at the JAEA gyrotron facility by means of IR thermography. The disk reaches thermal equilibrium and shows values consistent with the modeling. The microwave beam provided by a $40 \mathrm{~m}$ waveguide system with several mitre bends is not purely Gaussian. Also no arcing effects were observed. The higher order modes apparently cause parasitic heating effects. This is because they penetrate into the cavities neighboring the diamond disk and into the gap between the copper cuffs and the SS structure. The influence of the exact mode composition in the microwave beam is subject to further investigations.

Reference: T. A. Scherer, et al., Proc. EC-15 conf., Yosemite, USA 2008. 